International Journal of Wireless \& Mobile Networks (IJWMN) Vol. 3, No. 5, October 2011

\title{
ANalysis of THE Paging Costs IN Location MANAGEMENT FOR MOBILE COMMUNICATIONS NETWORKS LEVERAGING THE DIFFUSION CONSTANT
}

\author{
E. Martin and R. Bajcsy \\ Department of Electrical Engineering and Computer Science \\ University of California, Berkeley \\ California, USA \\ emartindeecs.berkeley.edu
}

\begin{abstract}
The recent growth of users in mobile communications networks has shown the need to efficiently manage the signalling burden related to Mobility Management. Within this field, the optimisation of Location Management costs has become a key research topic. In this sense, the development of accurate mobility models to describe the user's behaviour is still a pending task. In this article, having characterized the diffusion constant for distinct types of movements, the evaluation of the paging costs for different motion processes through the diffusion constant is reviewed, obtaining useful guidelines for their ranking in terms of signalling costs. As a consequence of this analysis, a novel parameter is introduced to account for how searchable a mobile user is, and whose inverse approximates the paging costs of a wide variety of motion processes.
\end{abstract}

\section{KEYWORDS}

Paging Costs, Diffusion Constant, Mobility Management, Location Management, Mobile Communications Networks

\section{INTRODUCTION}

Location Management is a topic of increasing importance due to the rise in the number of users in mobile communications networks [1-6]. Most of the recent research in this field has focused on the evaluation of the signalling costs involved in both location update and paging [7-10]. Common techniques to assess Location Management signalling costs make use of time-varying probability distributions on the mobile user's location, derived either from motion models or approximated by means of empirical data [11-17]. This strategy is especially suitable when the mobile terminal changes location according to stochastic processes. Considering for example an isotropic Brownian motion process with drift, the Gaussian probability density function for the location of the mobile can be applied [18], and the location probability can be obtained through the integration of the density function over the region of interest. For the particular analysis of the paging costs, an approach based on location probability distributions could be the information theoretical one, by means of relating the entropy of the distribution with the cost of paging, as the mean of the ordered distribution corresponds to the minimum paging costs [19]. However, this strategy faces the difficulty that the entropy and the mean number of locations paged can present very large variations. 
International Journal of Wireless \& Mobile Networks (IJWMN) Vol. 3, No. 5, October 2011

In this article, we examine the evaluation of the paging costs for different motion processes leveraging the diffusion constant. As a consequence of this analysis, we introduce a novel parameter to account for how searchable a mobile user is, and whose inverse approximates the paging costs of a wide variety of movements. The rest of this article is organized as follows: in Section 2 we analyze the behaviour of the diffusion constant for one-dimensional, twodimensional and planned movements. In Section 3, leveraging the diffusion constant to calculate the paging costs of different motion processes, we perform comparisons between them in order to obtain guidelines on their costs' ranking for different time intervals and mobility parameters. In Section 4 we introduce a novel parameter with the name Searchability Index, useful for the approximation of the paging costs. Conclusions are drawn in Section 5.

\section{STUDY OF THE DIFFUSION CONSTANT FOR DIFFERENT TYPES OF MOVEMENTS}

Considering isotropic Brownian motion process with drift, assuming movements of the user to the right or to the left with probability $p$ or $q$ respectively, the Gaussian probability density function for the location of the mobile can be applied [20], and two parameters with special importance in this type of processes can be leveraged for a deeper characterization of the motions: 1) the mean drift velocity $v$, defined as:

$$
v=(p-q) \frac{\Delta x}{\Delta t}
$$

and 2) the diffusion constant Dif :

$$
\text { Dif }=((1-p) p+(1-q) q+2 p q) \frac{(\Delta x)^{2}}{\Delta t}
$$

where $\Delta x$ and $\Delta t$ represent the space and time steps. Next we study the behaviour of the diffusion constant for different types of movements.

\subsection{Study of the diffusion constant for one-dimensional movements.}

A typical random movement where the mobile user can change direction randomly at each point can be analysed through the consideration of a Brownian motion process, with $p$ and $q$ taking values according to the type of movement. For example, for a one-dimensional movement along a straight line, if it is considered equally likely to move forward or backwards, then $p=q$ and 1$p-q$ is the probability to stay in the same point. However, $p$ and $q$ can take different values, thus reflecting certain preference forward or backward. In Figure 1 the variability of the diffusion constant with the mean velocity is shown for different values of $p$ and $q$.

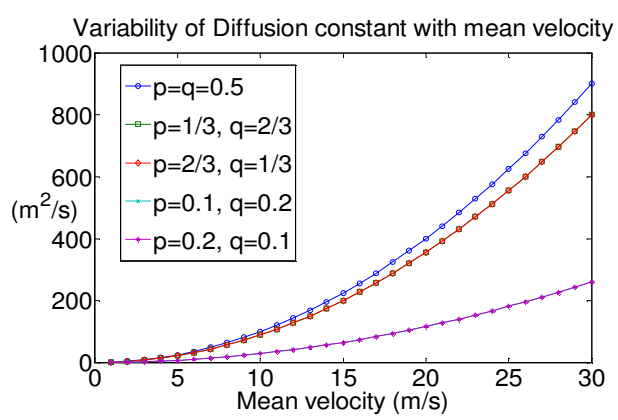

Figure 1. Variability of diffusion constant with mean velocity for one-dimensional random movement. 
In Figure 1, it can be seen that the diffusion constant increases parabolically with the mean velocity of the user. At the same time, the diffusion constant value does not vary if the $p$ and $q$ values are exchanged. Therefore, the diffusion constant reflects the mobility of the user, regardless of the direction of the movement. It is interesting to note that the sensors embedded in current state-of-the-art smart phones can be leveraged to obtain very precise information about the mobility of the user [12-17], which can be very helpful to manage the location of the mobile communications networks' users. Another clear consequence inferred from Figure 1 is the fact that as the $p$ or $q$ values increase, so does the diffusion constant, but for combinations of $\mathrm{p}$ and $\mathrm{q}$ which add up the same quantity, the diffusion constant will be higher for those values of $p$ and $q$ that enclose the larger area in a theoretical rectangle of dimensions $p \cdot q$.

\subsection{Study of the diffusion constant for two-dimensional movements.}

A two-dimensional movement where the user moves randomly towards any of four typical possible directions (North, South, East, West) at each point, can be split into two onedimensional movements with the same mobility characteristics, but half probabilities. That is, if the movement to any of the four possible directions is equally likely, $p$ and $q$ will be 0.25 for every one-dimensional version. In Figure 2, the influence of the addition of one more dimension for the movement in the diffusion constant is analysed.

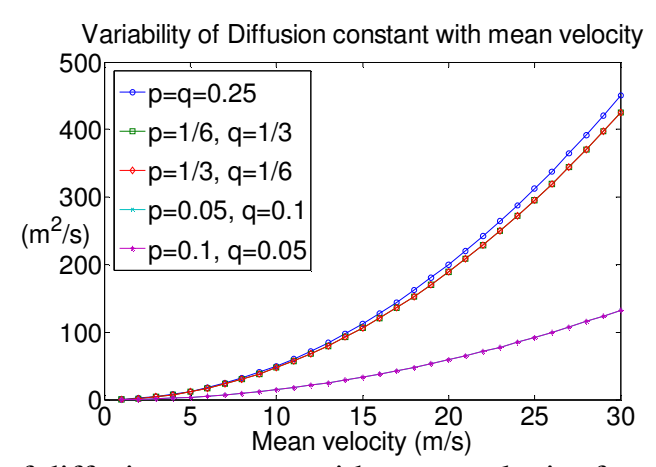

Figure 2. Variability of diffusion constant with mean velocity for two-dimensional random movement.

The diffusion constant for every one-dimensional version decreases approximately by half in comparison with the previous case, but the behaviour with the mean velocity and the $p$ and $q$ values is the same.

\subsection{Study of the diffusion constant for planned movements.}

The previous model can also be applied to situations such as those planned movements with constant speed and no random changes of direction, where theoretically the diffusion constant would be null. Carefully studying these movements [21, 22], taking into account the standard deviation in arrival time dev, the diffusion constant can be expressed as follows:

$$
\text { Dif }=\frac{2 \cdot(V \cdot d e v)^{2}}{\text { Duration }}
$$

Where Duration is the average trip duration and $V$ represents the average velocity. For example, for a planned trip of 2 hours, considering standard deviations in arrival time ranging 
International Journal of Wireless \& Mobile Networks (IJWMN) Vol. 3, No. 5, October 2011

from 1 minute to 10 minutes, the values that can be obtained for the diffusion constant are displayed in Figure 3.

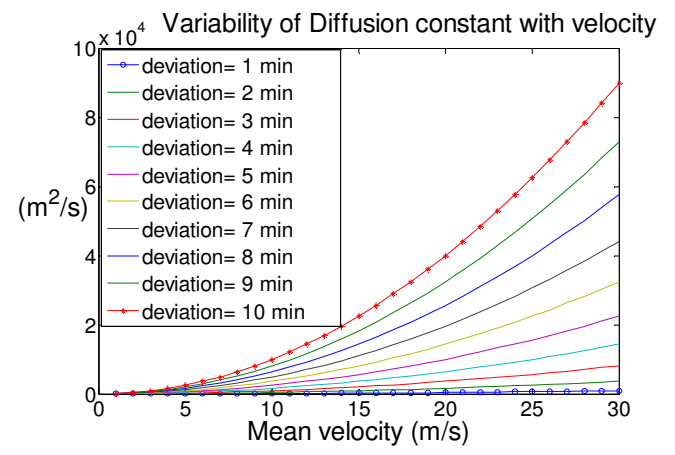

Figure 3. Variability of diffusion constant with mean velocity for planned movement.

It can be noticed the way in which the diffusion constant rises with the deviation in arrival times, and again, with the velocity. The increase of the diffusion constant with the value of the deviation is gradual. For higher velocities, such as those for high-speed trains or aircrafts, the values taken can be observed in Figure 4.

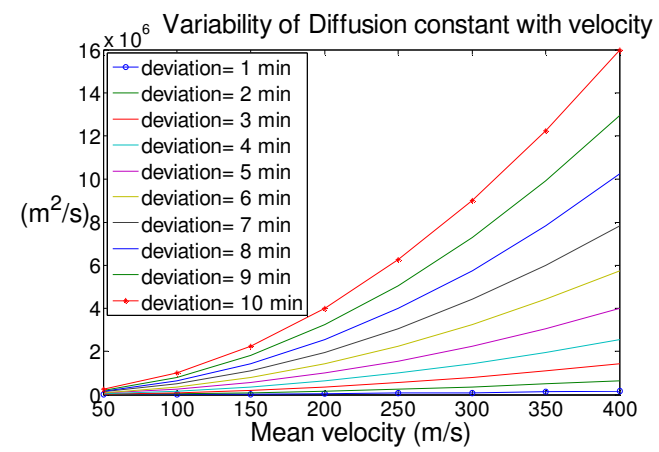

Figure 4. Variability of diffusion constant with mean velocity for planned movement and high velocity.

\section{APPLICATION OF THE DIFFUSION CONSTANT FOR THE CALCULATION OF THE PAGING COSTS.}

An approximation for the minimum of the paging costs by means of the diffusion constant is obtained in [23]: $(\text { Dif } \cdot t)^{\frac{n}{2}}$, where $t$ is the time elapsed since the last interaction mobile usernetwork, and $n$ is the movement dimension. In Figure 5, the variability of the paging costs for Brownian motions of various dimensions and for different call arrival rates is shown: 

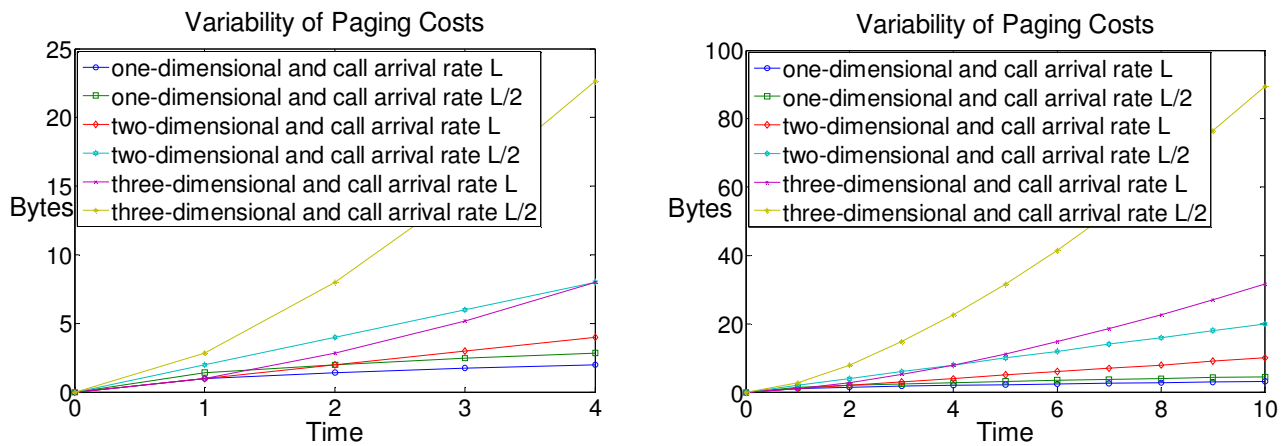

Figure 5. Variability of paging costs for different movement dimensions and call arrival rates, for short and long time intervals.

For simplicity purposes, the diffusion constant for the movements in Figure 5 has been taken as unity, and the time units are considered in terms of call inter-arrival periods. Comparing motion processes of different characteristics, for very short time intervals elapsed from the last interaction mobile user-network (typically a number of time units coincident with the value of the lower call-to-mobility ratio of the movements being compared, or one time unit in case that lower ratio is below unity), the paging costs for each particular call are very similar for all the different movement dimensions and call arrival rates, but the three-dimensional one with the lowest call arrival rate tends to outstand from the rest for presenting the fastest increase in time of the paging costs, with a parabolic evolution. The next movement with the highest paging costs is the two-dimensional one with the lowest call arrival rate, followed by the onedimensional one with the lowest call arrival rate. Consequently, at these early time intervals, although the higher the movement dimension, the larger the paging costs for processes with call-to-mobility ratios below unity (opposite behaviour for those processes with call-to-mobility ratios above unity), it is clearly noticed the key role played by the call arrival rate to classify the motion processes in terms of their paging costs: the lower the call arrival rate, the larger the paging costs for each particular call.

Still at very early stages, but for longer time intervals than in the previous considerations, the three-dimensional movement with high call arrival rate starts increasing its paging costs above those movements with lower dimensions and lower call arrival rates.

Comparing two motion processes with different call arrival rate, and no restrictions for their particular diffusion constants, we define a threshold value we name Dimensionality Threshold $\left(D_{T H}\right)$, measured in time units, and obtained by means of the following expression we derive in the Appendix I:

$$
D_{T H}=\frac{\left(C M R_{h}\right)^{3}}{\left(C M R_{l}\right)^{2}}
$$

Where $C M R_{h}$ and $C M R_{l}$ are the call-to-mobility ratios (high and low respectively) of the two types of processes being compared through their call arrival rates. The definition of this threshold is: 
Dimensionality Threshold: Length in time units over which the call arrival rate exchanges its role with the movement dimension as the key parameter to classify motion processes of different characteristics according to the value of their paging costs for each particular call.

As the time from the last interaction exceeds the Dimensionality Threshold, the threedimensional movement with low call arrival rate clearly becomes the one with the largest paging cost, followed by the three-dimensional one with high call arrival rates, next the twodimensional ones accordingly to their call arrival rates, and eventually the one-dimensional ones, again with larger paging costs for lower call arrival rates.

In Figure 6, the variability of the paging costs for Brownian motions of different dimensions and for different diffusion constants is shown:
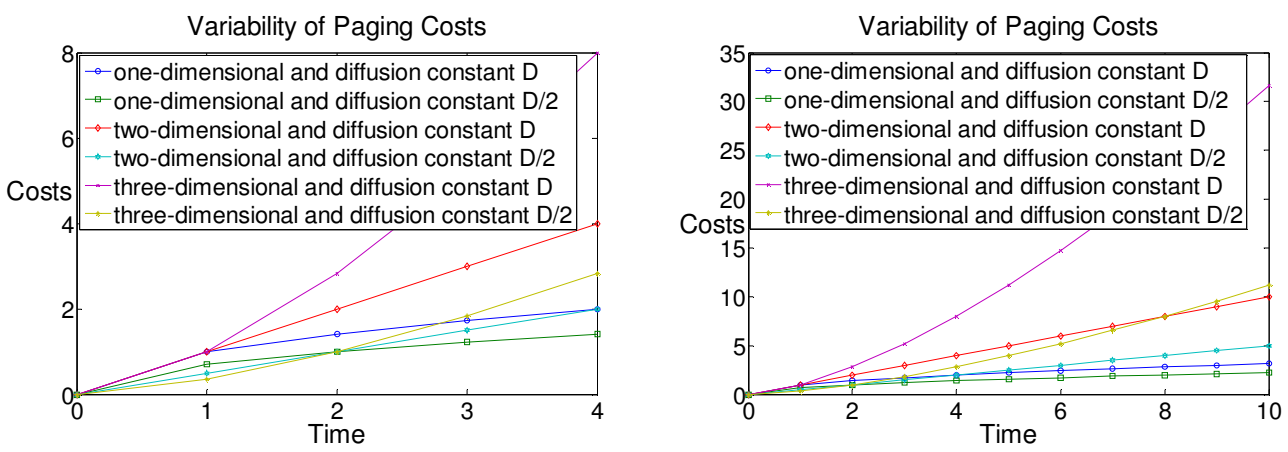

Figure 6. Variability of paging costs for different movement dimensions and diffusion constant values, for short and long time intervals.

For simplicity purposes, the call arrival rates for the motion processes in Figure 6 have been taken as unity. In order to perform the comparison related to the different movement characteristics, within the first of the shorter movement periods from the last interaction mobile user-network (although the same behaviour can be noticed within the first of the longer movement periods), the dimensionality of the movement has little importance to rank the paging costs. At this stage, the value of the diffusion constant rules the behaviour of the costs, and the higher the diffusion constant, the larger the paging costs. Actually, for these time intervals, in opposition to the behaviour of processes with call-to-mobility ratios below 1, for call-tomobility ratios above 1 it can be observed that the lower the movement dimension, the larger the paging costs. It is interesting to think about an explanation for this behaviour. The reasoning we propose is based on the fact that assuming a fixed amount of resources for a particular location area regardless of the movement dimensionality, the higher the movement dimension for diffusivities with a frequency lower than the call arrival rate, within the first movement period, the higher the mobile's specifiability in terms of amount of resources needed to track him down, therefore the lower that amount of resources, thus diminishing the paging costs for each particular call. Once the first movement period is passed, the higher the movement dimension, the higher the uncertainty of the next location of the mobile, thus the more dramatically the mobile's specifiability declines, therefore making the paging costs for each particular call larger. The basic idea of the previous statements is illustrated in a simplified way with Figure 7: 

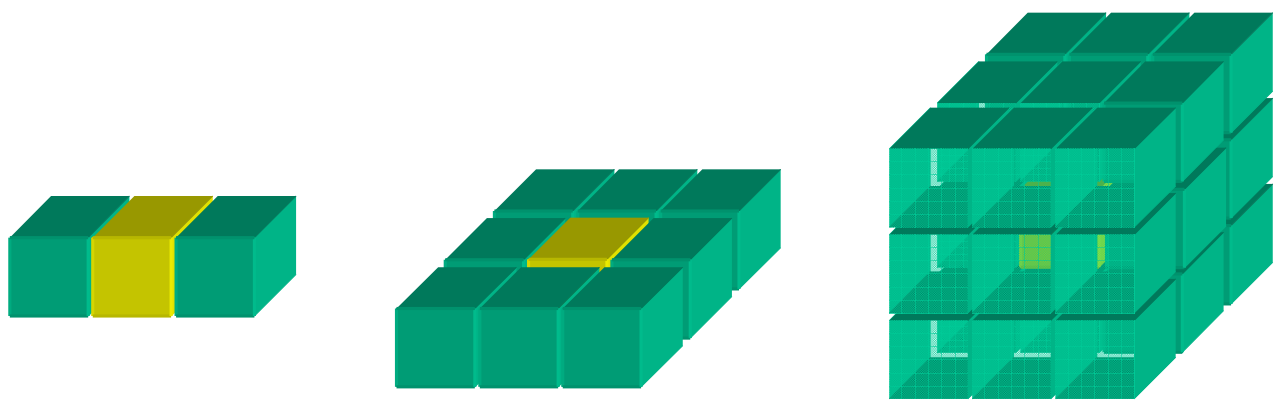

Figure 7. Variation of the mobile's specifiability with the movement dimension.

Assuming that the number of cells per location area increases with the movement dimension, when the movement frequency is lower than the paging frequency, within the first movement period, the amount of resources being used $\left(R_{s}\right)$ specifically to track down the mobile in the deployments represented in Figure 7 is:

$$
\left.R_{s}\right|_{t<T}=\left(\frac{1}{3^{n}}\right) \cdot R
$$

Where $R$ is the total amount of resources used for each location area, $n$ is the movement dimension, $t$ represents the time elapsed since the last interaction mobile-network, and $T$ is the movement period. As expected, $R_{s}$ falls when $n$ rises. On the other hand, once the first movement period is passed, the uncertainty for the whereabouts of the mobile increases with the movement dimension, and thus the amount of resources needed to track him down increases with the movement dimension, as shown by the new expression taken by $R_{s}$ :

$$
\left.R_{s}\right|_{t>T}=\left(\frac{3^{n}-1}{3^{n}}\right) \cdot R
$$

Graphically, assuming $R=1$ for simplicity purposes, the behaviour of $R_{s}$ with the movement dimension is presented in Figure 8:

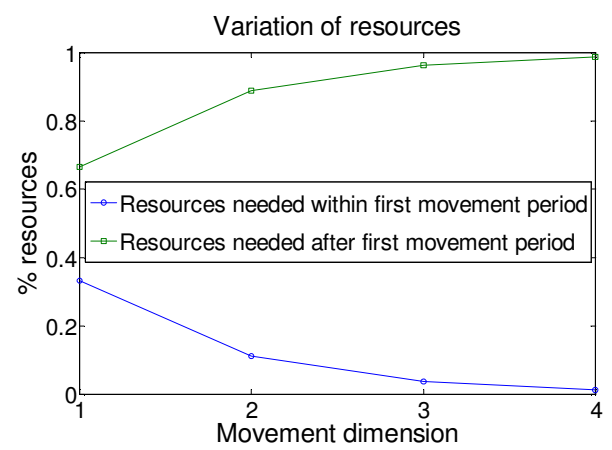

Figure 8. Variation of resources needed to track down a mobile with diffusivity frequency lower than call arrival rate, before and after the first movement period. 
As observed in Figure 8, the amount of resources needed varies with the movement dimension, in a way dependant on the time interval considered. It would be interesting to extend the previous reasoning beyond mobile communications networks users, and consider movements of entities in more than three dimensions. However we will not tackle that discussion in this article.

In Figure 9, the actual behaviour of the paging costs for each particular call for processes with call-to-mobility ratio above 1 and for time intervals within the first movement period are shown:

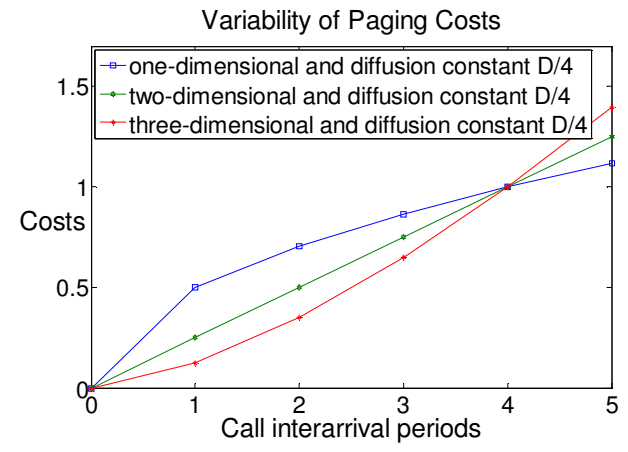

a) Diffusion constant $=1 / 4$

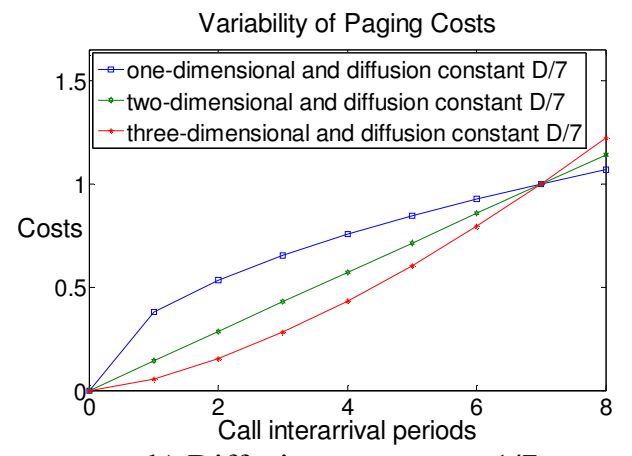

b) Diffusion constant $=1 / 7$

Figure 9. Variability of paging costs for different dimensions and diffusion constants providing a movement rate with longer period than the call inter-arrival one.

Continuing with the previous comparison between motion processes of different dimensions and diffusion constants, in between the first and the second movement period of the process with the highest call-to-mobility ratio of those being compared, although the diffusion constant remains as the main factor for the value of the paging costs, it starts becoming noticeable that the higher the movement dimension, the faster the paging costs increase with time.

For longer time intervals, the movement dimension keeps gaining importance regarding the value of the paging costs, and the diffusion constant continues loosing it, especially for one and two-dimensional movements. Making use of the Dimensionality Threshold again (in this case its definition will simply substitute the call arrival rate by the diffusion constant, while the mathematical formula remains the same), once the time elapsed from the last interaction usernetwork passes it, the paging costs of the motion processes are ranked firstly by their movement dimension (costs rise with it), and within each dimension, the higher the diffusion constant, the larger the paging costs become.

\section{SEARCHABILITY INDEX}

Given the importance of location information and context awareness in mobile computing [2428], next we leverage the results previously obtained for the behaviour of the paging costs with different process dimensions and call-to-mobility ratios, and we introduce a novel parameter we call Searchability Index $(S I)$, which will provide us with a good indication of how searchable a mobile user is. In other words, $S I$ will provide us with an indication of the granularity in the location information that the system has about the user, which will be very helpful for the network operators to optimize the Location Management procedures [2-6]. We have inferred the mathematical definition of the $S I$ from the results obtained analyzing multiple cases: 


$$
S I=T c /(P c)^{\left[\operatorname{Int}\left(\frac{D i f}{\lambda} \cdot t\right)\right]}
$$

With $t$ representing the time elapsed from the last interaction mobile user-network, the exponent of the denominator being the integer part of that time normalized to the value of the call-tomobility ratio, $P c$ symbolizing the number of possible new cells where the mobile can enter every time it moves, and $T c$ representing the total number of cells in its area of immediate influence. These two last quantities depend on the movement dimension. Defining a parameter we call "cluster one" $\left(C_{l}\right)$ as the numerical size of the area being administered in the onedimensional case (for example, number of cells per location area), $P c$ and $T c$ take the following expressions:

$$
\begin{aligned}
& P c=\left(C_{1}\right)^{n}-1 \\
& T c=\left(C_{1}\right)^{n}
\end{aligned}
$$

With $n$ representing the movement dimension. For simplicity purposes, we have chosen 3 as the reference value for $C_{1}$.

The meaning of the Searchability Index can be inferred from the fact that the larger its value, the more predictable the whereabouts of the mobile user are. Its values would range between 0 and $\infty$. The former for extremely unpredictable locations, and the latter for absolute certainty in the whereabouts of the mobile. The Searchability Index does not have units, and is an illustrative figure whose inverse approximates the value of the paging costs for each particular call for movements of different characteristics. In order to obtain a better approximation for the value of the costs over selected normalized time spans, we split the time into intervals of length unity by means of the integer part function.

The behaviour of the inverse of the Searchability Index is shown graphically in Figure 10:

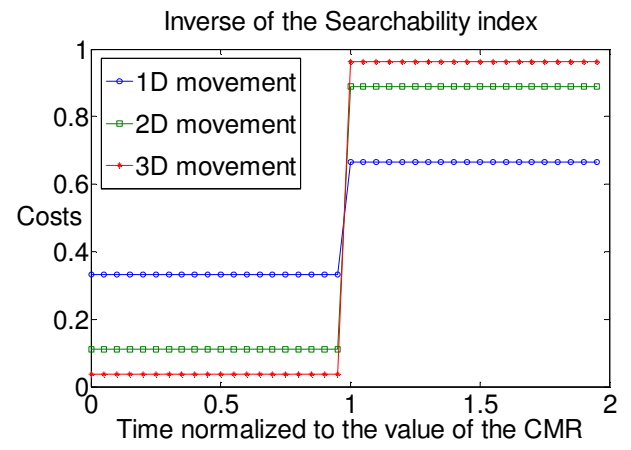

a) Around first time interval

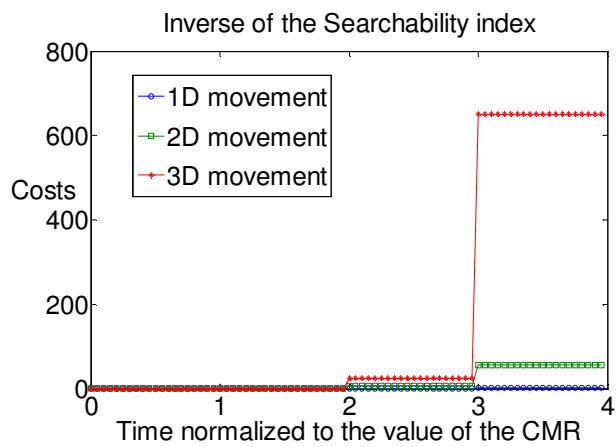

b) Longer time span

Figure 10. Evolution of the inverse of the Searchability Index.

However, through the study of series of average values of the paging costs and series of values of the inverse of the Searchability Index in the time intervals considered, a further refinement in the definition of the Searchability Index is possible in order to obtain a better approximation between both terms, by means of taking into account some more influences of parameters involved in the motion process. In this sense, we derive the following expression: 


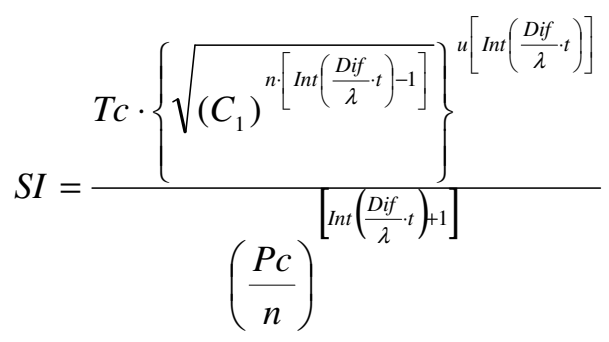

With $u(t)$ in the exponent of the numerator, defined as follows:

$$
u(t)= \begin{cases}0 & t<1 \\ 1 & t \geq 1\end{cases}
$$

And now, through the inverse of this refined Searchability Index, a better approximation for the value of the paging costs can be obtained. In Figure 11, the evolution of the inverse of this refined Searchability Index is shown for different normalized time lengths:
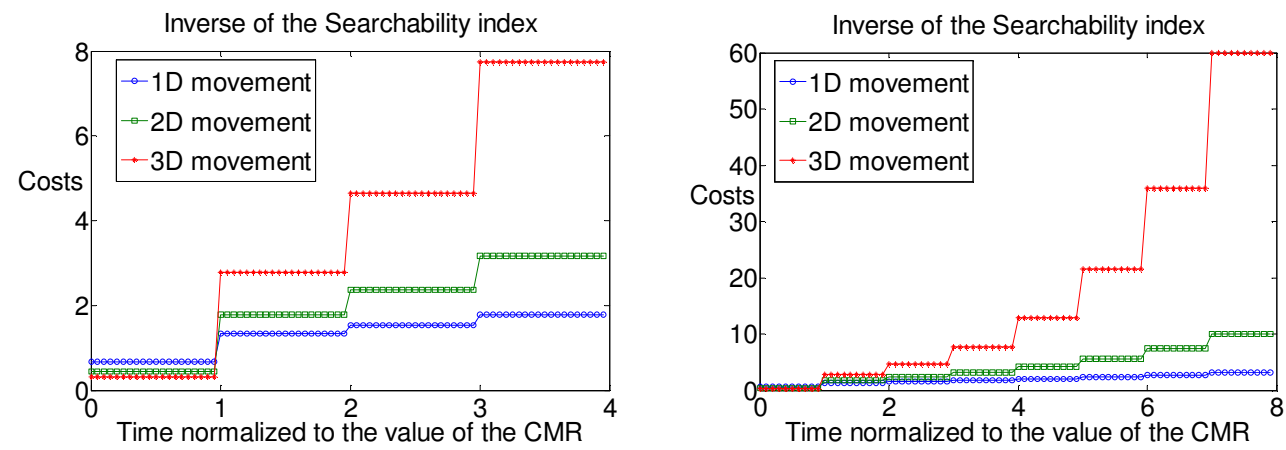

Figure 11. Evolution of the inverse of the refined Searchability Index, for short and long time intervals.

This Searchability Index accounts not only for the movement dimension and call-to-mobility ratio, but also for the network topology, in particular, the size of the clusters of cells. Its range of applicability through the formula we derived is: $C_{1}>2$, and length of the time in study below 10 normalized units, as otherwise, the results obtained would present higher values than the actual costs for the three-dimensional case. In Figure 12, a comparison between the actual paging costs and the inverse of the Searchability Index is shown for an interval of 4 time normalized units (average accuracies above 82\%, with larger values for one- and twodimensional movements): 


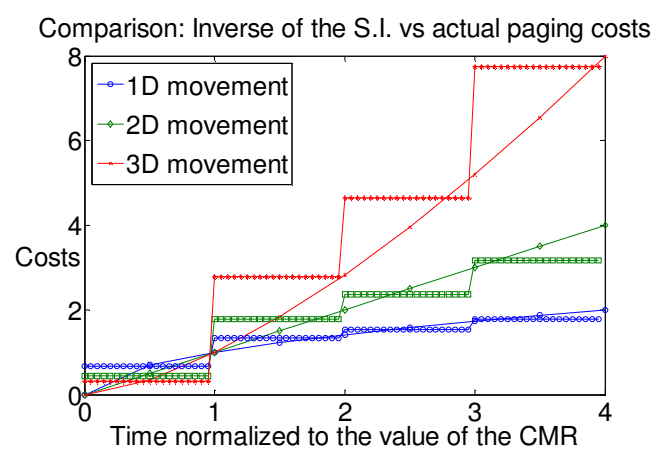

Figure 12. Comparison between the inverse of the Searchability Index and actual paging costs.

Additionally, Figure 12 shows that the inverse of the Searchability Index perfectly accounts for the exchange after the first normalized time unit between the movement dimensions in the ranking of more costly processes, when those processes have a call-to-mobility ratio above unity. The inverse of the Searchability Index also approximates correctly the paging costs for motion processes with call-to-mobility ratios below unity, for which the referred exchange between dimensions does not take place.

Having studied the behaviour of the inverse of the Searchability Index for different values of $C_{1}$ and time intervals, we can conclude that expectedly, the larger the value of $C_{1}$, the greater the resulting paging costs.

\section{Conclusions.}

The analysis of the diffusion constant shows that it accounts for the mobility of the user regardless of the direction of the movement. Applying the concept of the diffusion constant to obtain an approximation of the paging costs for Brownian movements of different dimensions, we reach the following conclusions: within the first units of the time elapsed from the last interaction mobile-network, although the higher the movement dimension, the larger the paging costs for processes with call-to-mobility ratios below unity (opposite behaviour for those processes with call-to-mobility ratios above unity), it is clearly noticed the key role played by the call arrival rate to rank the different movements; in fact, the lower the call arrival rate, the larger the paging costs for each particular call. Once the time elapsed from the last interaction mobile-network exceeds the Dimensionality Threshold, the movement dimension is the decisive parameter in the rise of the paging costs, and the call arrival rate takes a secondary role, especially for the one and two-dimensional movements. In particular, at these time intervals, the higher the dimensionality, the larger the paging costs, and for a same movement dimension, the lower the call arrival rate, the larger the paging costs for each call. An analogous study can be carried out in terms of the movement dimension and the diffusion constant, where the latter will show trends inverse to the call arrival rate.

In order to account for the behaviour of the paging costs of different motion processes, we have introduced a novel parameter called Searchability Index, whose meaning is based on the fact that the larger it becomes, the more predictable the whereabouts of the mobile user are, and its inverse approximates the value of the paging costs. The Searchability Index considers not only the dimensionality and call-to-mobility ratio of the process, but also the network topology, specifically, the size of the clusters of cells. It is important to notice that the inverse of the Searchability Index perfectly accounts for the exchange between the movement dimensions in the ranking of more costly movements after the first normalized time unit, for processes with 
International Journal of Wireless \& Mobile Networks (IJWMN) Vol. 3, No. 5, October 2011

call-to-mobility ratio above unity. The inverse of the Searchability Index is also consistent with motion processes whose call-to-mobility ratio is below unity and for which the referred exchange between dimensions does not take place.

\section{REFERENCES}

[1] H. Vijayakumar, M. Ravichandran, "Efficient location management of mobile node in wireless mobile ad-hoc network", Proceedings of National Conference on Innovations in Emerging Technology, NCOIET'11, p 77-84, 2011

[2] E. Martin, R. Bajcsy, "Savings in Location Management Costs Leveraging User Statistics", International Journal of Ubiquitous Computing, July, 2011.

[3] E. Martin, R. Bajcsy, "Variability of Location Management Costs with Different Mobilities and Timer Periods to Update Locations", International Journal of Computer Networks \& Communications, July, 2011.

[4] E. Martin, "A graphical Study of the Timer Based Method for Location Management with the Blocking Probability”, International Conference on Wireless Communications, Networking and Mobile Computing, 2011.

[5] E. Martin, "Characterization of the Costs Provided by the Timer-based Method in Location Management", International Conference on Wireless Communications, Networking and Mobile Computing, 2011.

[6] E. Martin, "New Algorithms to Obtain the Different Components of the Location Management Costs", International Conference on Wireless Communications, Networking and Mobile Computing, 2011.

[7] J. R. Gállego, M. Canales, A. Hernández-Solana, A. Valdovinos, “Adaptive paging schemes for group calls in mobile broadband cellular systems", IEEE International Symposium on Personal, Indoor and Mobile Radio Communications, PIMRC, p 2444-2449, 2010

[8] L. Jong-Hyouk, P. Sangheon, Y. Ilsun, C. Tai-Myoung, "Enabling a paging mechanism in network-based localized mobility management networks", Journal of Internet Technology, v 10, n 5, p 463-472, 2009

[9] G. Ashish, G. Navankur, K. Prakhar, "A speed based adaptive algorithm for reducing paging cost in cellular networks", Proceedings - 2009 2nd IEEE International Conference on Computer Science and Information Technology, ICCSIT 2009, p 22-25, 2009.

[10] V. Casares-Giner, P. García-Escalle, "A lookahead strategy for movement-based location update in wireless cellular networks", ITNG 2009 - 6th International Conference on Information Technology: New Generations, p 1171-1177, 2009.

[11] S. Madhavapeddy, K. Basu, A. Roberts, "Adaptive paging algorithms for cellular systems", IEEE Vehicular Technology Conference, 1995, v 2, p. 976-980.

[12] E. Martin, "Solving Training Issues in the Application of the Wavelet Transform to Precisely Analyze Human Body Acceleration Signals", IEEE International Conference on Bioinformatics and Biomedicine, 2010.

[13] E. Martin, R. Bajcsy, "Analysis of the Effect of Cognitive Load on Gait with off-the-shelf Accelerometers", International Conference on Advanced Cognitive Technologies and Applications, 2011.

[14] E. Martin, R. Bajcsy, "Considerations on Time Window Length for the Application of the Wavelet Transform to Analyze Human Body Accelerations", IEEE International Conference on Signal Processing Systems, 2011.

[15] E. Martin, “Optimized Gait Analysis Leveraging Wavelet Transform Coefficients from Body Acceleration”, International Conference on Bioinformatics and Biomedical Technology, 2011. 
International Journal of Wireless \& Mobile Networks (IJWMN) Vol. 3, No. 5, October 2011

[16] E. Martin, "Novel Method for Stride Length Estimation with Body Area Network Accelerometers", IEEE Conference on Biomedical Wireless Technologies, Networks, and Sensing Systems, 2011.

[17] E. Martin, "Real Time Patient's Gait Monitoring through Wireless Accelerometers with the Wavelet Transform", IEEE Conference on Biomedical Wireless Technologies, Networks, and Sensing Systems, 2011.

[18] C. Rose, R. Yates, "Location uncertainty in mobile networks: a theoretical framework" IEEE Communications Magazine, February 1997, p. 94-101.

[19] C. Rose, R. Yates, "Minimizing the average cost of paging under delay constraints". ACM wireless Networks, 1997, v1, n2, p. 211-219.

[20] A. Papoulis, "Probabilty, random variables, and stochastic processes". New York, McGraw-Hill, $3^{\text {rd }}$ edition, 1991.

[21] S. Rappaport, "Blocking, hand-off and traffic performance for cellular systems with mixed platforms" IKE Proceedings I, 1993, v. 140, n 5, p. 389.

[22] D. Kragic, A. Miller, P. Allen, "Real time tracking meets online grasp planning", Proceedings IEEE International Conference on Robotics and Automation, 2001, v 3, p 2460-2465

[23] C. Rose, "Minimizing the average cost of paging and registration: a timer-based method" Wireless Networks, 1996, v2, n2, p. 109-116.

[24] E. Martin, et al., "Enhancing Context Awareness with Activity Recognition and Radio Fingerprinting”, IEEE International Conference on Semantic Computing, 2011.

[25] E. Martin, et al., "Linking Computer Vision with off-the-shelf Accelerometry through Kinetic Energy for Precise Localization”, IEEE International Conference on Semantic Computing, 2011.

[26] E. Martin, T Lin, "Probabilistic Radio Fingerprinting Leveraging SQLite in Smart Phones", IEEE International Conference on Signal Processing Systems, 2011.

[27] E. Martin, R. Bajcsy, "Enhancements in Multimode Localization Accuracy Brought by a Smart Phone-Embedded Magnetometer", IEEE International Conference on Signal Processing Systems, 2011.

[28] E. Martin, "Multimode Radio Fingerprinting for Localization", IEEE Conference on Wireless Sensors and Sensor Networks, 2011.

\section{ApPendix I. MATHEMATICAL EXPRESSiOn FOR THE Dimensionality THRESHOLD.}

Considering a basic classification of motion processes for mobile communications networks users, based on the comparison of their call arrival rates, analyzing the behaviour of their paging costs for one, two and three dimensions of the movement, the point where the roles between the call arrival rate and the movement dimension are exchanged is located at the crossing of the costs curve of the two-dimensional one, characterized by low call arrival $\left(\lambda_{2}\right)$, with that of the three-dimensional one, characterized by high call arrival rate $\left(\lambda_{3}\right)$. Mathematically, the equation describing that crossing is:

$$
\left(\frac{\operatorname{Dif}_{2}}{\lambda_{2}} \cdot t\right)^{2 / 2}=\left(\frac{\operatorname{Dif}_{3}}{\lambda_{3}} \cdot t\right)^{3 / 2}
$$

From (12), obtaining the time solution: 


$$
t=\frac{\left(\frac{D i f_{2}}{\lambda_{2}}\right)^{2}}{\left(\frac{D i f_{3}}{\lambda_{3}}\right)^{3}}=\frac{\left(\frac{1}{C M R_{2}}\right)^{2}}{\left(\frac{1}{C M R_{3}}\right)^{3}}
$$

Therefore, naming that time interval as Dimensionality Threshold, the final expression is the following:

$$
D_{T H}=\frac{\left(C M R_{h}\right)^{3}}{\left(C M R_{l}\right)^{2}}
$$

\section{Authors}

E. Martin is carrying out research in the Department of Electrical Engineering and Computer Science at University of California, Berkeley. He holds a MS in Telecommunications Engineering from Spain and a $\mathrm{PhD}$ from England within the field of location management for mobile telecommunications networks. He has research experience in both industry and academia across Europe and USA, focusing on wireless communications, sensor networks, signal processing and localization.

R. Bajcsy received the Master's and Ph.D. degrees in electrical engineering from the Slovak Republic, and the Ph.D. in computer science from Stanford University, California. She is a Professor of Electrical Engineering and Computer Sciences at the University of California, Berkeley. Prior to joining Berkeley, she headed the Computer and Information Science and Engineering Directorate at the National Science Foundation. Dr. Bajcsy is a member of the National Academy of Engineering and the National Academy of Science Institute of Medicine as well as a Fellow of the Association for Computing Machinery (ACM) and the American Association for Artificial Intelligence. In 2001, she received the ACM/Association for the Advancement of Artificial Intelligence Allen Newell Award, and was named as one of the 50 most important women in science in the November 2002 issue of Discover Magazine. 